Original Paper http://ajol.info/index.php/ijbcs http://indexmedicus.afro.who.int

\title{
Qualités germinatives des graines et croissance des plantules de Isoberlinia spp. en milieu contrôlé (pépinière)
}

\author{
Pagadjovongo Adama SILUE*, Konan Édouard KOUASSI, \\ Kouadio Arsène Dieudonné KOFFI et Dodiomon SORO
}
Laboratoire de Botanique, UFR Biosciences, Université Félix HOUPHOUËT BOIGNY (Côte d'Ivoire), 22 BP 582 Abidjan 22, Côte d'Ivoire.
*Auteur correspondant ; E-mail : pagadsilue@gmail.com; Tél : (+225) 07791431.

\begin{abstract}
RESUME
Le genre Isoberlinia appartient à la famille des Caesalpiniaceae et comprend deux espèces forestières bien représentées dans les formations végétales du Nord de la Côte d'Ivoire. La présente étude vise à renforcer les informations de bases sur la domestication de ce genre. Deux milieux (milieu ombragé, milieu ouvert) abritant chacun des graines mises à germer suivant un dispositif en bloc randomisé à 100 unités expérimentales et des plantules issues de graines et de stumps ont été installés. Les observations de germination et des mesures sur la croissance des plantules y ont été régulièrement effectuées. Au terme des essais, le milieu ombragé a enregistré les meilleurs paramètres de germination avec un taux de germination de $96,80 \%$ et un temps de latence relativement court de 7 jours. En outre, la conservation des graines au réfrigérateur à $7{ }^{\circ} \mathrm{C}$ durant 5 semaines a permis de maintenir leur pouvoir germinatif alors que leur conservation à la température ambiante a entrainé une baisse d'au moins $60 \%$ de leur teneur en eau et une perte significative de leur pouvoir germinatif. Le sol à texture sableuse a permis d'obtenir les meilleurs paramètres de germination de même que les semis effectués à une profondeur inférieure à $3 \mathrm{~cm}$. La croissance des jeunes plants issus de graines et de stumps reste faible quel que soit le milieu comparativement à celle de certaines espèces savanicoles.
\end{abstract}

(C) 2017 International Formulae Group. All rights reserved.

Mots clés : Isoberlinia, paramètres de germination, graines récalcitrantes, Croissance des plantules, Côte d'Ivoire.

\section{Germinating qualities of seeds and seedlings growth of Isoberlinia spp. in a controlled environment (seedbed)}

\begin{abstract}
Isoberlinia spp. is a species of the family Caesalpiniaceae and a forest essence well represented in the plant formations of the North of Cote d'Ivoire. The target of this study is to evaluate the effect of abiotic factors on seed germination and the initial growth of seedlings of the genus Isoberlinia. Two environments (shaded
\end{abstract}


environment, open environment) sheltering each seed germinated according to a randomized block device with 100 experimental units and seedlings from seeds and stumps were installed. The germination observations were regularly carried out as well as growth measurements on the seedlings. The results show that the intensity of the light has a significant influence on the germination parameters with a higher germination rate $(96.80 \%)$ and a relatively short latency ( 7 days) in a shaded environment. When the seeds are stored at ambient temperature, their water content decreases by at least $60 \%$ after 3 weeks, leading to a significant loss of germinative power. Such seeds, called recalcitrant seeds, retain about $70 \%$ of their water content and present values of germination parameters close to those of freshly harvested seeds when stored in the refrigerator at $7{ }^{\circ} \mathrm{C}$ during 5 weeks. A soil with a sandy texture makes it possible to obtain the best germination results but the seedlings must be done at a depth of less than $3 \mathrm{~cm}$ at the risk of influencing the germination rate significantly. In addition, the study showed that the growth of young plants of Isoberlinia spp of the two types of experimentation is slow compared to that of certain savannah species.

(C) 2017 International Formulae Group. All rights reserved.

Keywords: Isoberlinia, germination parameters, recalcitrant seeds, growth of seedlings, Côte d'Ivoire.

\section{INTRODUCTION}

En zone soudanienne, les espèces ligneuses sont considérées comme sans intérêt comparativement aux grands arbres de la forêt équatoriale (Bamba, 2013). Cependant, une grande partie de ces espèces fournit aux populations rurales les produits indispensables à leur survie tels que les substances médicinales, les bois d'œuvre, le bois de feu, le fourrage (Ambé, 2001). L'exploitation incontrôlée des formations végétales boisées entraine la régression rapide, voire la disparition totale de certaines plantes très utiles aux communautés locales (Sanogo et al., 2006) alors que peu d'informations existent sur leur biologie et leur sylviculture. Parmi les principales espèces fréquemment exploitées dans le Nord de la Côte d'Ivoire, figurent Isoberlinia doka et Isoberlinia tomentosa qui sont des espèces bien inféodées aux zones tropicales arides, soudaniennes semi-arides et connues pour leur rusticité, leur pouvoir envahissant et recouvrant, leur croissance rapide (Bamba, 2013). Ces deux espèces concernées par cette étude sont les premières pourvoyeuses de bois d'œuvre dans le Nord du pays et dont les demandes sont de plus en plus fortes. Face à cette menace permanente, la domestication revêt une importance capitale pour la préservation de cette ressource phytogénétique. La production de plants de qualité constitue une garantie de réussite pour cette approche. Elle nécessite la mise en place d'un espace spécialisé telle que la pépinière qui constitue le lieu indiqué pour produire des plantules vigoureuses et de meilleure qualité (M'sadak et al., 2012 ; Nguema et al., 2014). Mais les mécanismes de germination des essences forestières dans les régions tropicales sont généralement inconnus. Il en est de même pour les délais de conservation des semences, de la cinétique de croissance et du développement des jeunes plants d'après Schwart et al. (2005). C'est pourquoi il a semblé important de porter une attention particulière à ces premières étapes du développement des plantules. La présente étude vise à renfoncer les bases de la sylviculture de Isoberlinia spp. D'une manière spécifique, il s'agit d'étudier l'effet de facteurs abiotiques (intensité lumineuse, types 
de sols, profondeur de semis et milieu de conservation) sur les qualités germinatives des graines et sur la croissance initiale des plantules afin de mieux maîtriser les premiers problèmes rencontrés lors des essais de reboisement. Cette étude tente aussi de résoudre le problème de conservation des graines de l'espèce dont le pouvoir germinatif baisse rapidement dans les conditions de conservation de la température ambiante (Bationo et al., 2005).

\section{MATERIEL ET METHODES}

\section{Zone d'étude}

Les essais ont été réalisés dans la localité de Kouto sur le site de la pépinière du cantonnement des eaux et forêts de ladite localité (Figure 1). Ce site est à la latitude $9^{\circ} 52^{\prime} 50^{\prime \prime}$ Nord et à la longitude $6^{\circ} 24^{\prime} 14^{\prime \prime}$ Ouest, sur une altitude de $363 \mathrm{~m}$. Le climat qualifié de soudanien est caractérisé par une longue saison sèche de novembre à mai et une courte saison des pluies de juin à octobre. La pluviométrie moyenne annuelle oscille entre 1000 et $1400 \mathrm{~mm}$. Les températures moyennes mensuelles sont comprises entre $25{ }^{\circ} \mathrm{C}$ et 31 ${ }^{\circ} \mathrm{C}$.

\section{Matériel végétal}

Dans le cadre des essais expérimentaux, deux types de matériel de reproduction ont été utilisés : les graines et les stumps (Figure 2). Les graines qui ont servi aux essais de germination ont été récoltées pendant les mois d'avril à juillet durant les années 2015 et 2016 sur des semenciers identifiés pour leur productivité et leur qualité de graines, dans le domaine rural. Les stumps sont des portions $(15-20 \mathrm{~cm})$ des plants âgés d'au moins un an (partie racinaire + tige) dont la partie aérienne a été supprimée au-dessus des premiers bourgeons et prélevés sur une pépinière sèche réalisée un an plutôt.

\section{Dispositif expérimental et méthodes d'expression des résultats}

Les essais expérimentaux ont été effectués sous l'effet de quatre (4) facteurs abiotiques. Il s'agit de l'intensité de l'ensoleillement, du milieu de conservation, de la profondeur de semis et des types de sols. La caractérisation de l'intensité lumineuse a nécessité la mise en place d'une ombrière constituée de claie recouverte de feuilles de palme et un dispositif à ciel ouvert correspondant respectivement au milieu ombragé et au milieu ouvert. La conservation des graines a été faite en milieu ambiant et dans un réfrigérateur à $7{ }^{\circ} \mathrm{C}$. Selon Bationo et al. (2005), les graines de Isoberlinia spp. perdaient leur pouvoir germinatif au bout de 5 semaines. Ainsi quatre catégories de graines ont fait l'objet d'essais: les graines fraichement récoltées (G1) considérées comme témoin, les graines conservées à la température ambiante pendant 3 semaines (G2), celles conservées à la température ambiante pendant 5 semaines (G3) et celles conservées au réfrigérateur pendant 5 semaines (G4). La profondeur de semis a été étudié en faisant des semis à cinq (5) niveaux de profondeurs $1 \mathrm{~cm} ; 2 \mathrm{~cm} ; 3 \mathrm{~cm} ; 5 \mathrm{~cm}$; et $7 \mathrm{~cm}$. Pour l'influence des sols sur la germination des graines, les essais ont été effectués sur les sols sableux, argileux et gravillonnaire qui correspondent aux principaux sols rencontrés dans les formations végétales naturelles de la région. Au cours des années 2015 et 2016, des expériences agronomiques ont été menées pendant cinq (5) 
mois en conditions naturelles d'éclairement, de température et d'hygrométrie. Les essais ont été conduits selon un dispositif en blocs randomisés, composés de 100 unités expérimentales. Les semis ont été effectués dans les sachets plastiques de polyéthylène $(18 \mathrm{~cm}$ de hauteur pour un diamètre de $10 \mathrm{~cm}$ ) perforés afin de drainer le milieu de culture. Les substrats utilisés ont été mis en sachets remplis jusqu'au bord supérieur. Une graine disposée à la surface du substrat cultural, est semé au centre dans un poquet profond de 1 $\mathrm{cm}$ environ. Le semis est précédé d'un arrosage des sachets 1 heure avant. Par la suite, les arrosages deviennent quotidiens dans les sachets disposés dans les différents milieux (milieu ouvert; milieu ombragé). Le comptage des graines germées a été effectué tous les jours pendant onze (11) jours et celui des graines saines non germées en fin d'expérience, afin d'évaluer et d'exprimer les paramètres de germination suivants :

- le temps de latence (TL) fait référence au temps écoulé de la date de semis à l'apparition des premières germinations ;

- le taux de germination (TG) est égal au rapport du nombre de graines germées $(\mathrm{G})$ à celui du nombre de graines semées $(\mathrm{N})$, multiplié par 100 ;

- l'énergie germinative (EG) est le pourcentage de semences (en nombre) qui germent jusqu'au moment de germination maximale, correspondant généralement à la période des 24 heures où se produit le plus grand nombre de germination ;

- la valeur culturale (VC) est l'expression de la combinaison du taux de germination (TG) et du taux de pureté (TP) (somme des semences germées et celles susceptibles de germer), divisée par 100 ;

- La teneur en eau des graines est la quantité d'eau contenue dans les graines et exprimé sous forme d'une fraction, en pourcentage, du poids de l'échantillon initial.

La croissance des plantules issues de graines et des pousses issues de rejets qui croissent en milieu ombragé et en milieu ouvert a été suivie durant cinq (5) mois. L'étude a consisté à mesurer la hauteur (croissance verticale) comprise entre le collet et le bourgeon terminal de chaque plante et le diamètre au collet (croissance horizontale) de 15 individus de plantules et de 15 individus de pousses choisies de façon aléatoire dans des lots de 100 individus de chaque catégorie de matériel végétal. Le paramètre calculé est la vitesse de croissance des plants qui est le nombre de centimètres acquis en 5 mois de croissance.

\section{Analyses des données}

Les moyennes des paramètres ont été comparées entre elles à l'aide de test $\mathrm{t}$ de Student en cas de normalité. En absence de normalité, le test de Mann-Withney a été appliqué. Ces deux tests ont été appliqués en présence de deux échantillons. En présence de plus de deux échantillons, le test ANOVA (en cas de normalité) a été utilisé et celui de Kruskal-Wallis (en cas d'absence de normalité) a été appliqué. Tous ces tests ont été réalisés grâce au logiciel XLSTAT 7.1. 

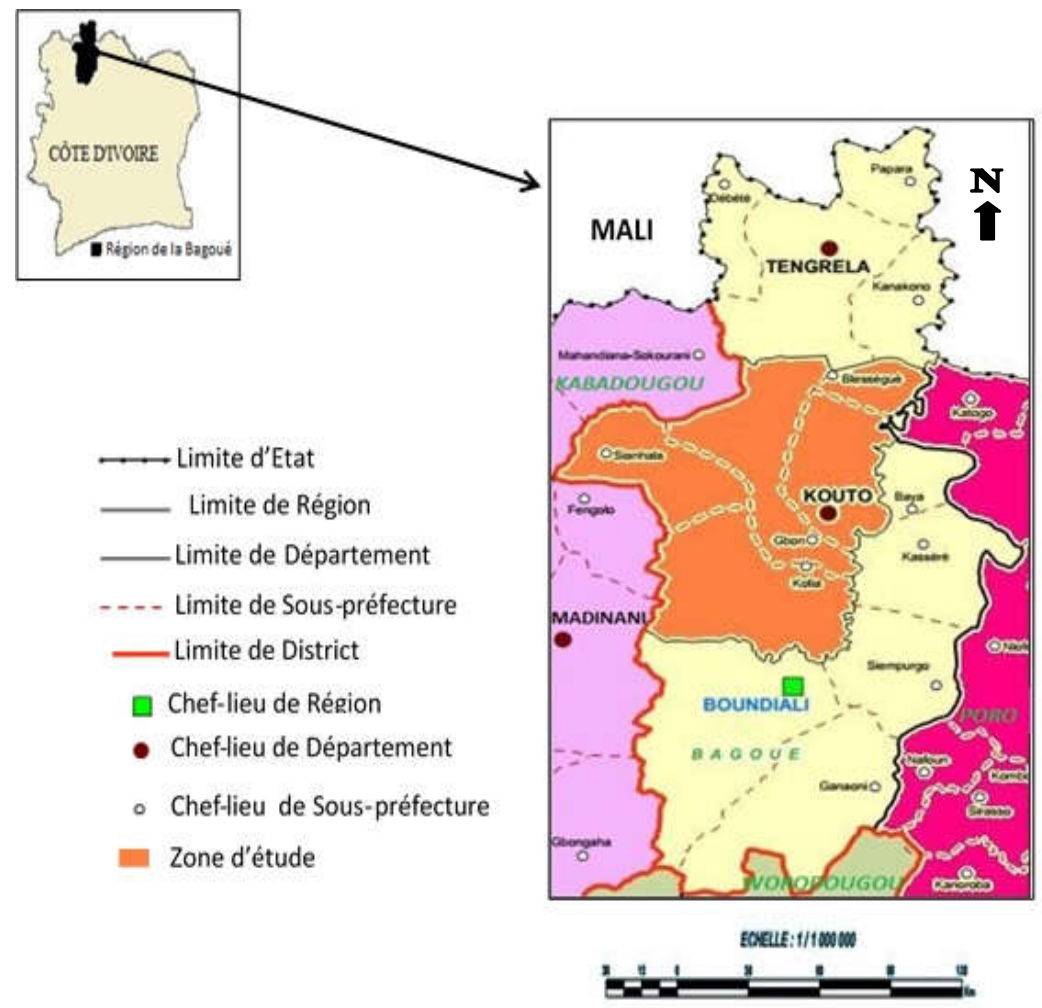

Figure 1 : Carte de localisation de la zone d'étude.

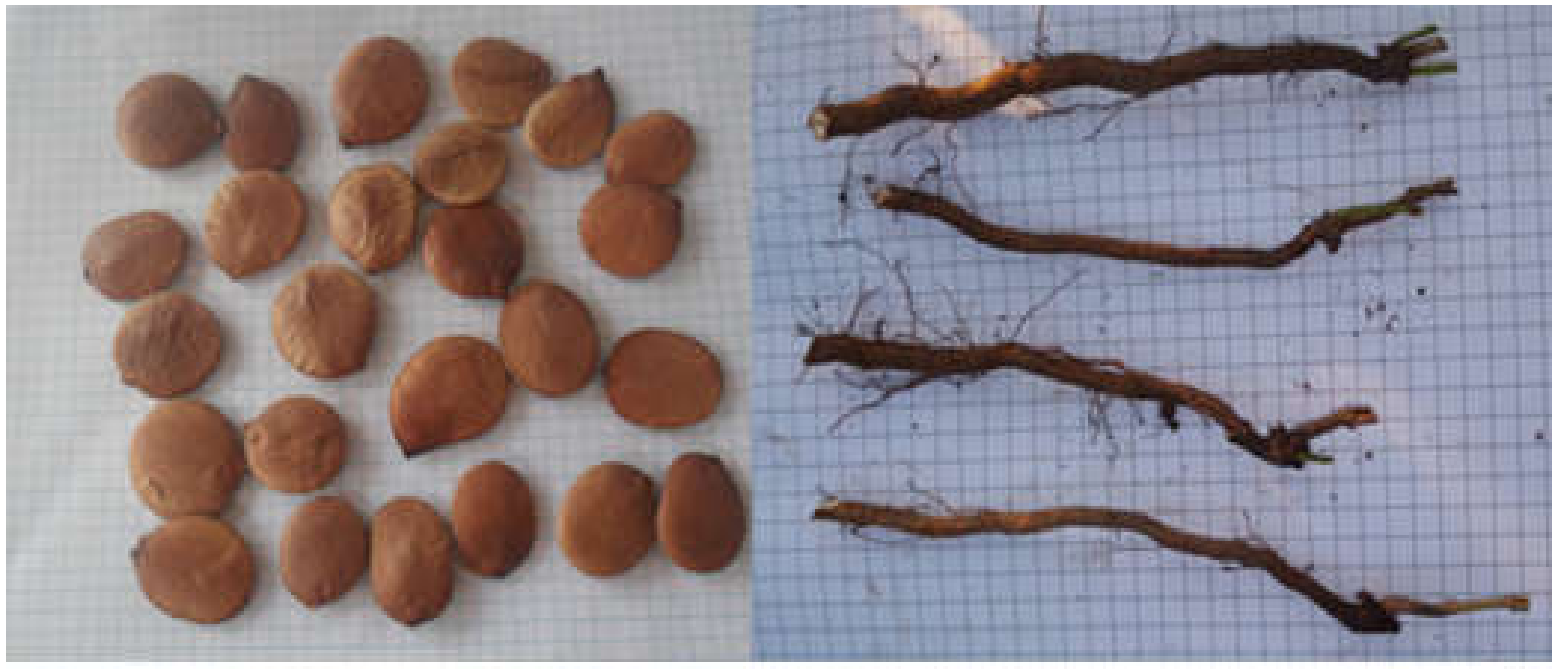

(a) Graines à maturité

(b) Stumps

Figure 2 : Types de semences de Isoberlinia spp. 


\section{RESULTATS}

\section{Influence de l'intensité d'ensoleillement sur les capacités germinatives des graines}

Les résultats concernant les paramètres de germination des graines de Isoberlinia spp. dans les deux milieux d'expérimentation sont présentés dans le Tableau 1. Le taux moyen de germination est plus élevé dans le milieu ombragé avec une valeur moyenne de $96,80 \%$. ( $t=-2,87 ; p=0,02)$. Le temps de latence des graines est le plus faible dans le milieu ombragé avec une valeur moyenne de 7 jours $(U=21 ; p=0,047)$. Pour les deux autres paramètres que sont l'énergie germinative et la valeur culturale, les résultats ont montré que les fortes valeurs moyennes de l'énergie germinative et de la valeur culturale ont été enregistrées dans le milieu ombragé avec des valeurs respectivement de $51,8 \%$ et $93,75 \%$. $(U=3,5 ; p=0,07)$.

\section{Influence de la durée et du milieu de conservation sur le pouvoir germinatif Influence sur la teneur en eau des graines}

Les moyennes des valeurs de la teneur en eau des graines de Isoberlinia spp. fraichement collectées sous les semenciers et soumis aux différentes formes de stockage se résument dans le Tableau 2. La catégorie G1 a une teneur en eau d'environ $15 \%$. Globalement, les pertes en eau des graines selon les modes de conservation oscillent entre $25,68 \%$ et $87,46 \%$ de la teneur en eau de la catégorie G1. La conservation des graines à la température ambiante influence significativement leur teneur en eau $\left(\chi^{2}=\right.$ $17,30 ; p<0,0001)$. La catégorie G4 perd moins d'eau et conserve au moins $70 \%$ de sa teneur en eau du poids frais. Le test comparatif des moyennes des teneurs en eau de G4 et de G1 n'a pas de différences significatives $\left(\chi^{2}=17,31 ; \mathrm{p}=0,22\right)$. Par contre on note un effet significatif du milieu de conservation $\left(\chi^{2}=17,31 ; p=0,0004\right)$ entre
G4 et G2 d'une part et un effet très significatif $\left(\chi^{2}=17,31 ; p<0,0001\right)$ entre G4 et G3 d'autre part sur les moyennes des teneurs en eau.

\section{Influence sur les paramètres de germination} des différentes catégories de graines

Les résultats de la comparaison des paramètres de germination des graines en fonction de la durée et du mode de conservation sont consignés dans le Tableau 3. La catégorie G1 présente les meilleures valeurs des paramètres de germination par rapport aux trois autres catégories que sont G2, G3 et G4. La comparaison des valeurs des moyennes montre qu'il existe une différence très significative entre la catégorie témoin (G1) et les autres catégories $\left(\chi^{2}=17,9 ; \mathrm{p}<\right.$ 0,0001). A la température ambiante, les graines perdent partiellement leur pouvoir germinatif au bout de 3 semaines de conservation. Les paramètres de germination sont ainsi influencés dans des proportions de l'ordre de $45 \%$. La conservation des graines à la température ambiante durant 5 semaines est fatale au pouvoir germinatif des graines. On enregistre avec ces dernières un temps de latence (18 jours) qui est supérieur au double de celui des graines de 1 jours ( 7 jours) et des valeurs des autres paramètres presque nulles. Il y a une différence significative entre les valeurs moyennes des paramètres de germinations des catégories $\mathrm{G} 2$ et $\mathrm{G} 3(\mathrm{U}=$ $0,00 ; p=0,009)$. Par contre, les graines de la catégorie $\mathrm{G} 4$, gardent leur pouvoir germinatif dans des proportions de l'ordre de $80 \%$ et donnent des valeurs des paramètres de germination supérieures à celles de la catégorie G2. Il y a une différence significative entre les catégories G2 et G4 pour les valeurs moyennes des paramètres de germination ( $t=-6,75 ; p=0,0001)$ sauf pour le temps de latence $(U=19,5 ; p=0,15)$. Les valeurs des paramètres de germination de la catégorie G4 sont proches de celles de la catégorie G1 (témoin) bien qu'il existe des 
différences statistiques entre les moyennes des paramètres $(\mathrm{t}=4,56 ; \mathrm{p}=0,002)$.

\section{Influence de la profondeur de semis sur les capacités germinatives}

Les résultats obtenus au cours de ces essais montrent clairement que la profondeur influence la germination chez Isoberlinia spp. Les meilleurs résultats des paramètres de germination ont été obtenus pour des profondeurs comprises entre $1 \mathrm{~cm}$ et $2 \mathrm{~cm}$ (Tableau 4).

Pour cet intervalle de profondeur de semis, on obtient des taux de germination supérieurs à $90 \%$ et les différences observées entre les paramètres de germinations ne sont pas significatives $(\mathrm{t}=1,71 ; \mathrm{p}=0,12)$ sauf pour le temps de latence $(\mathrm{t}=-5,77 ; \mathrm{p}=$ $0,0004)$. Au-delà de cet intervalle, à $3 \mathrm{~cm}$ de profondeur de semis les valeurs des paramètres de germination chutent d'au moins $30 \%$ à l'instar du taux de germination qui est de $61,40 \%\left(\chi^{2}=9,96 ; p=0,0009 / U=3,77\right.$; $\mathrm{p}<0,0001)$. A $5 \mathrm{~cm}$ et plus on note une chute des valeurs des paramètres de germination d'au moins $50 \%$. On obtient des taux de germination inférieurs à $50 \%$ et des temps de latence qui sont le double de ceux de l'intervalle $1 \mathrm{~cm}$ et $2 \mathrm{~cm}$. On note une différence significative de la profondeur de semis à partir de $5 \mathrm{~cm}$ sur les paramètres de germination $\left(\chi^{2}=22,16 ; \mathrm{p}<0,0001\right)$.

\section{Influence des types de sols sur la germination.}

Le Tableau 5 présente les paramètres de germination des graines dans chacun des sols. Le sol sableux induit de meilleures possibilités de germination (83\%) par rapport aux deux autres sols utilisés, avec $56,2 \%$ et $29,8 \%$ respectivement pour les sols argileux et gravillonnaires $(\mathrm{F}=3,77 ; \mathrm{p}<0,0001)$. En outre le temps de latence est relativement court sur les sols sableux et argileux (10 jours) par rapport au sol gravillonnaire (12 jours) $\chi^{2}$ $=10,29 ; p=0,0016)$. En ce qui concerne l'énergie germinative, le sol gravillonnaire présente la plus petite valeur par rapport aux deux autres sols $(F=3,77 ; p=0,0004)$. Les valeurs moyennes de l'énergie germinative entre les sols sableux et argileux sont égales $(\mathrm{F}=3,77 ; \mathrm{p}=0,20)$. Pour la valeur culturale, le sol sableux enregistre la plus forte valeur $(70,91 \%)$ par rapport aux sols argileux $(34,34 \%)$ et au sol gravillonnaire $(9,83 \%)$. Les différences entre les moyennes de la valeur culturale sont très significatives $\left(\chi^{2}=10,29 ; p\right.$ $<0,0001)$.

\section{Croissance des plantules issues de graines et des pousses issues de rejet en fonction de l'intensité de la lumière}

Les résultats des mesures de hauteurs et de diamètres des plantules sont présentés dans le Tableau 6. La croissance en hauteur issues des graines, dès le premier mois de levée diffère significativement pour les deux milieux étudiés $(t=6,47 ; p<0,0001)$, elle atteint en moyenne $6,54 \mathrm{~cm}$ en milieu ombragé et seulement $4,09 \mathrm{~cm}$ en milieu ouvert. Au terme des 5 mois d'observation, la situation s'équilibre et la croissance moyenne des plantules en milieu ouvert $(8,02 \mathrm{~cm})$ devient semblable à celle des plantules en milieu ombragé $(8,35 \mathrm{~cm})(\mathrm{t}=0,41 ; \mathrm{p}=0,68)$ avec des vitesses moyennes de croissance de $36,13 \mathrm{~mm} /$ mois en milieu ombragé et 78,67 $\mathrm{mm} /$ mois en milieu ouvert. Les différences entre ces moyennes sont significatives $(\mathrm{U}=$ $50,00 ; p=0,008)$. En ce qui concerne la croissance en hauteur des pousses issues des stumps, aucune différence significative n'a été notée pour les deux milieux dès le premier mois $(t=1,43 ; p=0,17)$. Cette valeur atteint en moyenne $2,74 \mathrm{~cm}$ en milieu ombragé et $2,26 \mathrm{~cm}$ en milieu ouvert. Au bout de 5 mois de croissance la situation reste toujours équilibrée et la croissance moyenne demeure 
faible quel que soit le milieu ( $\mathrm{t}=1,43 ; \mathrm{p}=$ $0,45)$ avec des vitesses moyennes de 20,18 $\mathrm{mm}$ en milieu ombragé et $23,45 \mathrm{~mm}$ en milieu ouvert. Les différences entre les moyennes des vitesses de croissance ne sont pas significatives $(U=50 ; p=0,37)$.

L'étude comparée de la croissance verticale des plantules et des pousses montre que les premières croissent plus vite que les secondes avec des valeurs moyennes d'élongation hautement significatives quel que soit le milieu $(\mathrm{t}=7,53 ; \mathrm{p}<0,0001)$.

Pour la croissance diamétrale (Figure 3 ), on note une différence significative entre les deux milieux dès le premier mois pour les plantules issues des graines $(U=39,50 ; p=$
0,0005). La croissance moyenne en milieu ouvert est supérieure à celle du milieu ombragé avec des valeurs respectives de 27,33 $\mathrm{mm}$ et de $21 \mathrm{~mm}$. Pour les pousses, des différences significatives sont également observées au niveau de la croissance diamétrale $(U=27 ; p=0,01)$. Elle atteint en moyenne $19,09 \mathrm{~mm}$ en milieu ouvert contre 14,09 $\mathrm{mm}$ en milieu ombragé. L'étude comparée de la croissance en diamètre entre les plantules et les pousses montre que les plantules ont une bonne croissance que les pousses avec des différences significatives au niveau des valeurs moyennes que l'on soit en milieu ombragé $(U=147 ; p=0,0002)$ ou en milieu ouvert $(U=139 ; p=0,0006)$.

Tableau 1 : Moyennes des principaux paramètres de germination de Isoberlinia spp. en fonction de l'intensité d'ensoleillement.

\begin{tabular}{lcc}
\cline { 2 - 3 } & Milieu ombragé & Milieu ouvert \\
\hline TL (jours) & $7,20 \pm 1,64$ & $9,20 \pm 1,09$ \\
TG (\%) & $96,80 \pm 2,38$ & $81,40 \pm 11,74$ \\
EG (\%) & $51,80 \pm 10,87$ & $46,80 \pm 20,56$ \\
VC (\%) & $93,75 \pm 4,63$ & $68,48 \pm 15,43$ \\
\hline
\end{tabular}

TL : Temps de Latence ; TG : Taux de germination ; EG : Energie germinative ; VC : Valeur Culturale.

Tableau 2 : Moyennes de la teneur en eau du poids frais et du pourcentage en eau des différentes catégories de graines de d'Isoberlinia spp.

Teneur en eau du poids frais $(\%)$

\begin{tabular}{lcc}
\hline G1 & $15,15 \pm 0,54^{\mathrm{a}}$ & 100 \\
$\mathrm{G} 2$ & $5,80 \pm 1,33^{\mathrm{b}}$ & 38,28 \\
$\mathrm{G} 3$ & $1,90 \pm 0,98^{\mathrm{c}}$ & 12,54 \\
$\mathrm{G} 4$ & $11,26 \pm 2,68^{\mathrm{a}}$ & 74,32 \\
\hline
\end{tabular}

G1 : graines fraichement récoltées; G2 : graines conservées durant 3 semaines à la température ambiante; G3 : graines conservées durant 5 semaines à la température ambiante; G4 : graines conservées au réfrigérateur à $7{ }^{\circ} \mathrm{C}$ durant semaines. NB : sur chaque ligne il n'y a pas de différence significative entre les moyennes suivie d'une même lettre au seuil de $\alpha=$ 0,05 . 
Tableau 3 : Moyennes des principaux paramètres de germination des graines de Isoberlinia spp. en fonction de la durée et du milieu de conservation.

\section{Catégories de graines}

\begin{tabular}{lcccc}
\hline & G1 & G2 & G3 & G4 \\
\hline TL (jours) & $7,20 \pm 1,64^{\mathrm{a}}$ & $10,40 \pm 0,89^{\mathrm{a}}$ & $18,00 \pm 1,41^{\mathrm{c}}$ & $9,50 \pm 0,58^{\mathrm{a}}$ \\
TG (\%) & $96,80 \pm 2,39^{\mathrm{a}}$ & $52,40 \pm 9,71^{\mathrm{c}}$ & $1,00 \pm 1,41^{\mathrm{d}}$ & $89,75 \pm 2,63^{\mathrm{b}}$ \\
EG (\%) & $51,80 \pm 10,87^{\mathrm{a}}$ & $21,20 \pm 7,60^{\mathrm{c}}$ & $2,50 \pm 0,71^{\mathrm{d}}$ & $36,25 \pm 5,50^{\mathrm{b}}$ \\
VC (\%) & $93,75 \pm 4,63^{\mathrm{a}}$ & $34,98 \pm 14,67^{\mathrm{b}}$ & $0,04 \pm 0,07^{\mathrm{d}}$ & $80,60 \pm 4,67^{\mathrm{b}}$ \\
\hline
\end{tabular}

TL : Temps de Latence ; TG : Taux de germination; EG : Energie germinative ; VC : Valeur Culturale.

G1 : graines fraichement récoltées; G2 : graines conservées durant 3 semaines à la température ambiante ; G3 : graines conservées durant 5 semaines à la température ambiante ; G4 : graines conservées au réfrigérateur à $7{ }^{\circ} \mathrm{C}$ durant semaines. NB : sur chaque ligne il n'y a pas de différence significative entre les moyennes suivie d'une même lettre au seuil de $\alpha=$ 0,05 .

Tableau 4 : Moyennes des principaux paramètres de germination des graines de Isoberlinia spp. en fonction de la profondeur de semis.

\begin{tabular}{lccccc} 
& \multicolumn{5}{c}{ Profondeur de semis } \\
\cline { 2 - 6 } & $\mathbf{1 ~ C m}$ & $\mathbf{2 ~ C m}$ & $\mathbf{3 ~ C m}$ & $\mathbf{5 ~ C m}$ & $\mathbf{7 ~ C m}$ \\
\hline TL (jours) & $8,40 \pm 0,55^{\mathrm{a}}$ & $10,40 \pm 0,55^{\mathrm{b}}$ & $13,00 \pm 1,00^{\mathrm{c}}$ & $17,20 \pm 0,45^{\mathrm{d}}$ & $16,60 \pm 0,55^{\mathrm{d}}$ \\
TG (\%) & $93,00 \pm 3,32^{\mathrm{a}}$ & $90,60 \pm 4,56^{\mathrm{a}}$ & $61,40 \pm 4,67^{\mathrm{b}}$ & $44,00 \pm 3,08^{\mathrm{c}}$ & $21,00 \pm 1,58^{\mathrm{d}}$ \\
EG (\%) & $59,40 \pm 9,63^{\mathrm{a}}$ & $46,00 \pm 14,61^{\mathrm{ab}}$ & $39,20 \pm 16,84^{\mathrm{bc}}$ & $27,40 \pm 8,29^{\mathrm{c}}$ & $20,60 \pm 1,67^{\mathrm{c}}$ \\
VC (\%) & $88,06 \pm 6,14^{\mathrm{a}}$ & $85,58 \pm 6,17^{\mathrm{a}}$ & $41,97 \pm 4,84^{\mathrm{b}}$ & $22,60 \pm 4,10^{\mathrm{c}}$ & $6,62 \pm 0,83^{\mathrm{d}}$ \\
\hline
\end{tabular}

TL : Temps de Latence ; TG : Taux de germination ; EG : Energie germinative ; VC : Valeur Culturale.

NB : sur chaque ligne il n'y a pas de différence significative entre les moyennes suivie d'une même lettre au seuil de $\alpha=$ 0,05 .

Tableau 5 : Moyennes des principaux paramètres de germination des graines de Isoberlinia spp. en fonction des types de sols.

\begin{tabular}{lccc} 
& Sol sableux & Sol argileux & Sol gravillonnaire \\
\hline TL (jours) & $10,40 \pm 0,55^{\mathrm{a}}$ & $10,40 \pm 0,55^{\mathrm{a}}$ & $12,40 \pm 0,55^{\mathrm{b}}$ \\
TG (\%) & $83,00 \pm 1,67^{\mathrm{a}}$ & $56,20 \pm 1,92^{\mathrm{b}}$ & $29,80 \pm 8,31^{\mathrm{c}}$ \\
EG (\%) & $56,20 \pm 5,26^{\mathrm{a}}$ & $46,40 \pm 0,88^{\mathrm{ab}}$ & $26,20 \pm 8,61^{\mathrm{b}}$ \\
VC (\%) & $70,91 \pm 2,79^{\mathrm{a}}$ & $34,34 \pm 4,11^{\mathrm{b}}$ & $9,83 \pm 4,01^{\mathrm{c}}$ \\
\hline
\end{tabular}

TL : Temps de Latence ; TG : Taux de germination ; EG : Energie germinative ; VC : Valeur Culturale.

NB : sur chaque ligne il n'y a pas de différence significative entre les moyennes suivie d'une même lettre au seuil de $\alpha=$ 0,05 . 
Tableau 6 : Moyennes des paramètres de croissance des jeunes tiges de Isoberlinia spp. en fonction des milieux ombragés et ouverts.

\begin{tabular}{llcc} 
& $\begin{array}{l}\text { Origines des } \\
\text { tigelles }\end{array}$ & Milieu ombragé & Milieu ouvert \\
\hline Hauteur totale moyenne & graines & $6,54 \pm 1,28$ & $4,09 \pm 0,72$ \\
initiale $(\mathrm{cm})$ & Stumps & $2,74 \pm 0,88$ & $2,26 \pm 0,65$ \\
\hline Hauteur totale moyenne & graines & $8,35 \pm 1,85$ & $8,02 \pm 1,85$ \\
finale $(\mathrm{cm})$ & Stumps & $3,74 \pm 0,94$ & $3,43 \pm 0,96$ \\
\hline Vitesse de croissance & graines & $36,13 \pm 0,30$ & $78,67 \pm 0,51$ \\
$(\mathrm{~mm} /$ mois $)$ & Stumps & $20,19 \pm 0,17$ & $23,45 \pm 0,20$ \\
\hline Diamètre moyen au collet & graines & $21,00 \pm 0,03$ & $27,33 \pm 0,05$ \\
$(\mathrm{~mm})$ & Stumps & $14,09 \pm 0,05$ & $19,09 \pm 0,02$ \\
\hline
\end{tabular}

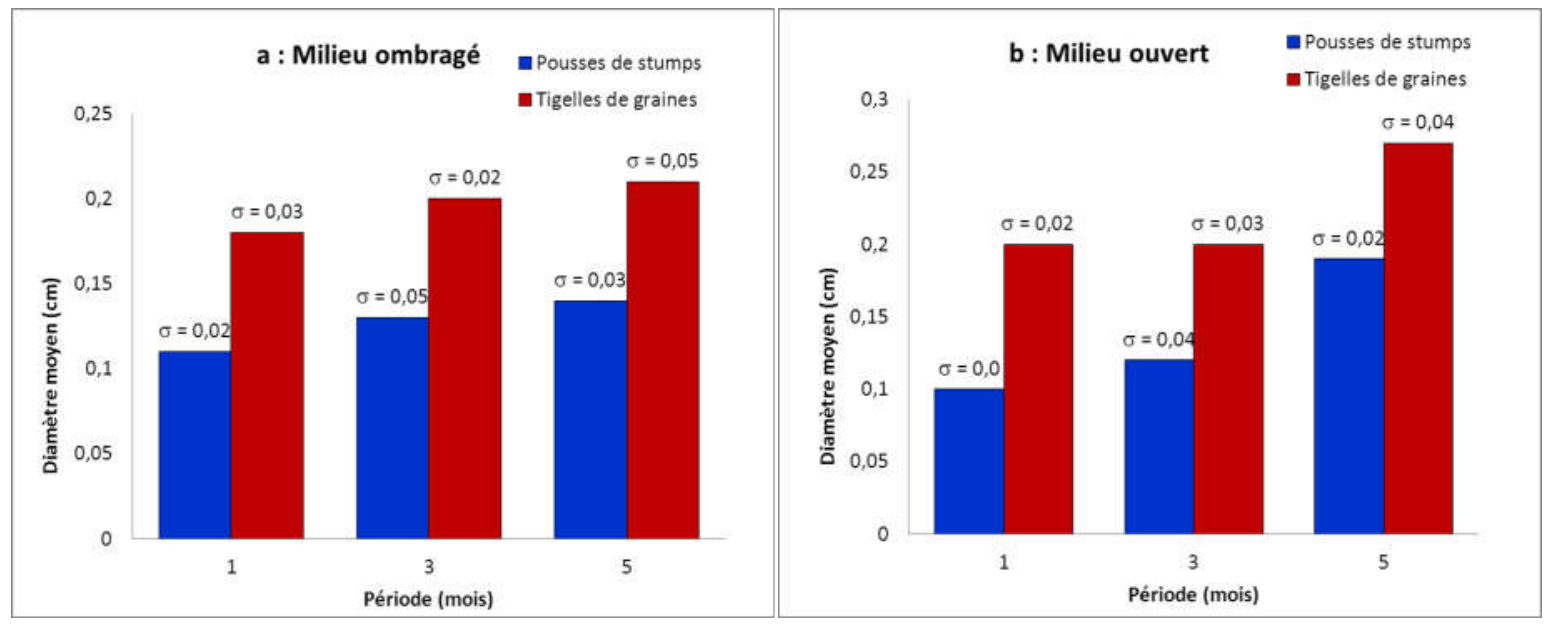

Figure 3 : Croissance en diamètre des plantules issues des graines et des pousses issues des stumps de Isoberlinia spp. en milieux ombragé (a) et ouvert (b).

\section{DISCUSSION}

Influence de l'intensité d'ensoleillement sur les paramètres de germination des graines

Cette étude a montré que pour avoir une germination rapide et en grande quantité des graines de Isoberlinia spp. en pépinière, il faut les semer en milieu ombragé. Les graines semées en milieu ombragé ont un temps de latence relativement court et un taux de germination élevée que celles semées en milieu ensoleillé. Des études similaires réalisées par Kouadio et al. (2014) à l'Est de la Côte d'Ivoire ont montré que l'intensité d'éclairage modérée influençait positivement le délai et le taux de germination de Entandrophragma angolense (Meliaceae). L'auteur souligne que les phytochromes localisés dans les semences ont parfois besoin d'une intensité lumineuse modérée pour faciliter la sortie des radicules. Les délais courts et les taux de germination élevés confirment ceux observés au Burkina Faso 
où, au laboratoire les temps de latence variant entre 2 et 4 jours et un taux de germination de $100 \%$ ont été notés (Bationo et al., 2005). Par contre ces résultats sont supérieurs à ceux obtenus par Yemadje (2004) avec un temps de latence de 18 jours et un taux de germination de $26 \%$. Cette différence pourrait s'expliquer par plusieurs facteurs que sont le type de substrat utilisé pour l'essai ou probablement le bon état sanitaire des graines semées. En effet, les graines de Isoberlinia spp. sont souvent attaquées par des larves endocarpiques d'insectes. En ce qui concerne l'énergie germinative, paramètre qui renseigne un peu sur la survie et la mortalité des plantules après le stade de germination, le milieu ombragé affecte mieux ce paramètre. En effet, dans ce milieu, plus de $50 \%$ des graines viables germent pour une période énergétique relativement courte ( 9 à 12 jours). Ces bonnes potentialités germinatives (énergie germinative élevée et période germinative courte) dont disposent les graines en milieu ombragé pourront faciliter et encourager la production de plants en pépinière. En effet, les semences qui germent lentement et irrégulièrement dans les conditions favorables de laboratoire risquent de donner de gros déchets et des plantules peu vigoureux sur le terrain et ceux d'autant plus que les conditions de température et d'humidité sont peu propices (Touré, 2001). Pour déterminer la qualité d'un lot de semences, certains auteurs utilisent le terme de valeur culturale. C'est un critère précis de comparaison de lots de semences d'une même espèce. Dans cette étude, les valeurs culturales obtenues dans les différents milieux d'étude sont supérieures à $60 \%$. Ce qui montre la bonne capacité germinative des graines de Isoberlinia spp. indépendamment de l'intensité de l'ensoleillement dans les conditions de cette étude. Cependant, la valeur culturale fortement élevée $(\geq 90 \%)$ dans le milieu ombragé montre qu'il faudra y utiliser une quantité plus réduite de graines qu'en milieu ouvert pour avoir un semis pas trop clair (Touré, 2001).

\section{Influence du milieu et de la durée de conservation sur le pouvoir germinatif des graines}

L'évaluation des caractéristiques germinatives des graines fraichement récoltées a mis en évidence une très forte capacité germinative des graines $(\geq 95 \%)$ à l'image des résultats obtenus par Bationo et al. (2005). Cette forte capacité germinative est acquise avec des graines dont la teneur en eau est de l'ordre de 15\%. L'étude sur les caractéristiques germinatives des lots de graines a montré que la baisse des valeurs des paramètres de germination est progressive au fur et à mesure que la conservation des graines est longue. Une baisse de la teneur en eau ( $\geq 60 \%$ ) entraine systématiquement une baisse des capacités germinatives des graines. Dès lors, on peut affirmer comme Bationo et al. (2005) que les graines de Isoberlinia spp. sont effectivement récalcitrantes. En effet, pour les graines récalcitrantes, une réduction de la teneur en eau au-delà d'un seuil relativement élevé entraine malheureusement leur mort. Toutefois, à l'issue de l'étude, les résultats obtenus mettent en évidence le rôle capital de la température dans le maintien du pouvoir germinatif des graines. En effet la conservation des graines au réfrigérateur à 7 ${ }^{\circ} \mathrm{C}$ pendant 5 semaines permet d'obtenir des performances germinatives proches de celles des graines fraichement récoltées, suite au maintien de leur teneur en eau à au moins $70 \%$. Ces résultats corroborent ceux de Agyili et al. (2007) qui ont signalé que les graines de Garcinia kola perdaient leur capacité germinative dès que leur teneur en eau devient inférieur à $30 \%$. Ils sont également similaires à ceux de Jiofack et Dondjang (2007) qui ont remarqué que les diaspores de Tetracarpidium conophorum (Euphorbiaceae) conservés à la température de $4{ }^{\circ} \mathrm{C}$ pendant un mois atteignaient un taux de germination de $83,3 \%$. 
Influence de la profondeur de semis sur les paramètres de germination des graines

On observe que les graines mises à l'épreuve de la profondeur de semis ont un comportement différent. Les résultats traduisent une décroissance régulière des paramètres de germination à partir de $3 \mathrm{~cm}$ de profondeur de semis. Les semis effectués entre $1 \mathrm{~cm}$ et $2 \mathrm{~cm}$ de profondeur permettent de maintenir les paramètres de germination à des proportions élevées. Les fortes fluctuations des paramètres germinatifs observées au-delà de $2 \mathrm{~cm}$ de profondeur pourraient être dues au mode de germination des graines de Isoberlinia spp. En effet, selon Bationo et al. (2005), l'émergence de la tigelle chez la graine de cette espèce se fait lorsque l'un des cotylédons reste plaqué contre le sol par mucilage et que l'autre hors contact avec le sol («cotylédon supérieur») pivote progressivement pour libérer la gemmule.

\section{Influence du type de sols sur les paramètres de germination des graines}

Concernant le comportement des graines de Isoberlinia spp. vis-à-vis du type de sols, cette étude montre qu'elles ont une très bonne aptitude à la germination sur sol sableux. Les données des paramètres de germination sont intermédiaires sur sol argileux et plus faibles sur sol gravillonnaire. Touré (2001) rapporte que le sable permet un bon contact entre les semences et la source d'humidité. Mais cette propriété du sable ne peut être la raison suffisante des résultats obtenus car des essais de germination de Daniella oliveri (Caesalpiniaceae) sur des sols à textures similaires à celles de cette étude n'ont pas montré de différences significatives sur les capacités germinatives des graines de cette espèce (Houehounha et al., 2009). L'hypothèse plausible serait à rechercher au niveau de la germination cryptogé des graines. Ce type de germination est une adaptation du grand groupe hypogé où la tigelle n'émerge qu'après que la radicule est atteinte 10 à 15 $\mathrm{cm}$ de profondeur dans le sol (Bationo et al., 2005).

Croissance des tigelles issues de graines et des pousses issues de rejet de stumps en fonction de l'intensité de la lumière

Après un mois de vie, les jeunes plants de Isoberlinia spp. qu'ils soient issues de graines ou de rejets de stumps, présentent une dynamique de croissance verticale meilleure dans le milieu ombragé par rapport au milieu ouvert. Mais au bout de cinq mois d'observation, les taux de croissance des jeunes plants issus de graines sont plus élevés que ceux issus des stumps (rejets). Ces jeunes plants issus de graines sont plus vigoureux avec un système foliaire plus dense. Des travaux faits au Centre du Benin, sur Daniella oliveri ont prouvé que les plants issus de la multiplication végétative croissent moins vite que ceux issus de graines (Houehounha et al., 2009). Les stumps de Isoberlinia ne présentent pas un avantage de croissance par rapport au plantules issues des graines.

Par ailleurs, la croissance des plants, quel que soit le type de matériel végétal, est plus lente comparée à d'autres espèces de savanes. C'est le cas de Guiera senegalensis (Combretaceae) qui présente une hauteur moyenne de l'ordre de $25 \mathrm{~cm}$ et un diamètre moyen de l'ordre de $3,22 \mathrm{~mm}$ et de Guibourtia ehie (Caesalpiniaceae) dont les valeurs moyennes sont de l'ordre de $17,18 \mathrm{~cm}$ pour la hauteur et de $2,20 \mathrm{~mm}$ pour le diamètre (Amani et al., 2015 ; Kouadio et al., 2013). Cette croissance lente également observée chez les plantules de Terminalia avicennioides (Combretaceae) a été qualifiée de rythmique. C'est une croissance caractérisée par des phases de repos temporaires ou de faible croissance (Bationo et al., 2010).

\section{Conclusion}

L'étude réalisée sur la germination des graines de Isoberlinia spp. a montré qu'elles possèdent de bonnes potentialités 
germinatives. Les meilleurs paramètres de germination des graines de l'espèce ont été obtenus en milieu ombragé. La germination est très rapide chez cette Caesalpiniaceae mais l'influence de certains facteurs abiotiques qui sont soient extrinsèques (types de sol, profondeur de semis, température) ou intrinsèques (teneur en eau) ralentissent cette capacité germinative. Pour ce dernier cas, les graines de l'espèce ne germent plus dès que leur teneur en eau (rapportée au poids frais) devient faible $(<80 \%)$. Les températures basses permettent de maintenir le pouvoir germinatif des graines dans des proportions proches de celles des graines fraichement récoltées. Le suivi des paramètres de croissance initiale des jeunes plants a montré une croissance lente, comparativement à d'autres espèces savanicoles. Néanmoins, cette dynamique de croissance est influencée positivement par le milieu ombragé. La photosensibilité semble être un facteur responsable de la croissance lente de cette espèce. Un renforcement des informations scientifiques générées par cette étude permettra à terme d'améliorer les connaissances sur la multiplication végétative de cette espèce savanicole à multiples usages, d'amorcer une sylviculture artificielle et rationnelle et de gérer durablement cette ressource utile.

\section{CONFLIT D'INTERETS}

Les auteurs déclarent qu'ils n'ont pas de conflits d'intérêts.

\section{CONTRIBUTIONS DES AUTEURS}

SPA a conduit les travaux de terrain, l'analyse et le traitement des données collectées et la rédaction du manuscrit. KKE a participé à la mise en place du dispositif expérimental, la discussion des résultats et la correction du manuscrit. KKAD a contribué aux traitements statistiques des données, à la discussion des résultats et à la rédaction du manuscrit. SD a apporté un appui à la discussion des résultats et des corrections au manuscrit.

\section{REMERCIEMENTS}

Nous adressons nos remerciements aux Agents du Cantonnement des Eaux et Forêts du Département de Kouto de nous avoir aidé dans la réalisation de cette étude.

\section{REFERENCES}

Agyili J, Sacande M, Koff E, Peprah T. 2007. Improving the collection and germination of West African Garciniakola Heckel seeds. New Forests, 34: 269-279. DOI: http://link.springer.com/article/10.1007/s 11056-007-9054-7

Amani A, Dan Guimbo MM, Mahamane A, Saadou M, Lykke AM. 2015. Germination et croissance de quatre espèces de Combretaceae en pépinière. Tropicultura, 33(2): 135-145. DOI : http://tropicultura.org/text/v33n2

Ambé GA. 2001. Les fruits sauvages comestibles des savanes guinéennes de Côte-d'Ivoire : état de la connaissance par une population locale, les Malinké. Biotechnol. Agron. Soc. Environ., 5(1): 43-58.

DOI :https://science.report/pub/2432076 1

Bamba N. 2013. Etude morphologique, phénologique et ethnobotanique des espèces des genres Isoberlinia et Berlinia dans le village de San au Nord de la Côte d'Ivoire. Mémoire DEA, Université Nangui Abrogoua, Côte d'Ivoire, p.60.

Bationo BA, Ouedraogo SJ, Somé AN, Pallo F, Boussim IJ. 2005. Régénération naturelle d'Isoberlinia doka Craib. Et Stapf. Dans la forêt classée du Nazinon (Burkina Faso). Cahiers Agricultures, 14(3): DOI :http://revues.cirad.fr/index.php/cah iers-agricultures/article/view/30522 
Bationo BA, Some AN, Ouedraogo SJ, Kalinganire A. 2010. Croissance comparée des plantules de cinq espèces ligneuses soudaniennes élevées en rhizotron. Sécheresse, 21(3): 196-202. DOI: http://10.1684/sec.2010.0255

Houehounha R, Avohou HT, Sinsin B, Tandiepkon AM. 2009. Approches de régénération artificielle de Daniella oliveri (Rolfe) Hutchison et Dalziel. Int. J. Biol. Chem. Sci., 3(1): 7-19. DOI: http://readcube.com/articles/10.4314/ijbc s.v3i1.42730

Jiofack RBT, Dondjang JP. 2007. Caractérisation et étude de la germination des diaspores de Tetracarpidium conophorum (Müll. Arg.) Hutch. Et Dalz. Int. J. Biol. Chem. Sci., 1(2): 136-142. DOI : http://dx.doi.org/10.4314/ijbcs.v1i2.3968 3

Kouadio K, Koné M, Soro D. 2014. Influence de l'ensoleillement sur la germination de quatre essences forestières en voie d'extinction dans les forêts ivoiriennes. J. Ani. \& Plan. Sci., 23(1): 3529-3538. DOI :

http://www.m.elewa.org/JAPS/2014/23( 1)

Kouadio K, Dibi HN, Bomisso L, Ettien RBK. 2013. Effet de l'intensité d'ensoleillement sur la croissance initiale en pépinière de Guibourtia ehie (A. Chev.) Leonard (Caesalpiniaceae), espèce couramment exploitée et menacée d'extinction, dans 1'Unité de Gestion Forestière de Bossematié (Côte d'Ivoire). Int. J. Biol. Chem. Sci., 7(6): 2292-2300. DOI :http://dx.doi.org/10.4314/ijbcs.v7i6 .10

M'sadak Y, Elouaer MA, Dhahri M. 2012. Croissance comparée des plantes de gombo en culture de pleine terre selon les modes semis direct et repiquage. Algerian Journal of Arid Environment, 2(2): 62-70. DOI : http://dspace.univouargla.dz/jspui/handle/123456789/6540

Nguema NP, Ondo-Azi AS, Mouele BJ, Ntsame NRL, Souza A. 2014. Effet de la composition de différents substrats culturaux sur quelques paramètres de croissance de Gambeya lacourtiana De Wild en pépinière au nord-est du Gabon. J. Appl. Biosci., 73: 5902-5910. DOI : http://ajol.info/index.php/jab/article/view $/ 111643$

Sanogo S, Sanogo AK, Yossi H. 2006. Collecte et conservation durable des graines et d'échantillons de plantes. Rapport de recherche de la campagne 2005/2006. Bamako : 12e session de la Commission Scientifique de l'Institut d'Économie Rurale (IER).

Schwartz C, Muller JC, Decroux J. 2005. Guide de la fertilisation raisonnée. Editions La France Agricole: Paris.

Touré Y. 2001. Etude des potentialités agroforestières, de la multiplication et des usages de Pterocarpus erinaceus Poir. en zone soudanienne du Burkina Faso. Mémoire d'Ingénieur du Développement Rural, Université Polytechnique de Bobodioulasso, Burkina Faso, p. 120.

Yemadje RG. 2004. Contribution à la gestion durable des forêts au Bénin : Biodiversité des endomycorhizes d'Isoberlinia doka (Craib \& Stapf) dans les différentes formations végétales de la forêt classée de Wari-maro (Nord Bénin). Mémoire d'Ingénieur Agronome FSA, Université d'Abomey Calavi, Bénin, p.127. 